\title{
Metamorphosis of the Mushroom Bodies; Large-Scale Rearrangements of the Neural Substrates for Associative Learning and Memory in Drosophila
}

J. Douglas Armstrong, ${ }^{1,4}$ J. Steven de Belle, ${ }^{2,3}$ Zongsheng Wang, ${ }^{1}$ and Kim Kaiser ${ }^{1}$

${ }^{1}$ Division of Molecular Genetics

University of Glasgow

Glasgow G11 6NU, UK

${ }^{2}$ Max-Plank-Institut für Biologische Kybemetik

D-72076 Tübingen, Germany

\begin{abstract}
Paired brain centers known as mushroom bodies are key features of the circuitry for insect associative learning, especially when evoked by olfactory cues. Mushroom bodies have an embryonic origin, and unlike most other brain structures they exhibit developmental continuity, being prominent components of both the larval and the adult CNS. Here, we use cell-type-specific markers, provided by the P\{GAL4\} enhancer trap system, to follow specific subsets of mushroom body intrinsic and extrinsic neurons from the larval to the adult stage. We find marked structural differences between the larval and adult mushroom bodies, arising as the consequence of large-scale reorganization during metamorphosis. Extensive, though incomplete, degradation of the larval structure is followed by establishment of adult specific $\alpha$ and $\beta$ lobes. Kenyon cells of embryonic origin, by contrast, were found to project selectively to the adult $\gamma$ lobe. We propose that the $\gamma$ lobe stores information of relevance to both developmental stages, whereas the $\alpha$ and $\beta$ lobes have uniquely adult roles.
\end{abstract}

${ }^{3}$ Present address: Department of Biological Sciences, University of Nevada, Las Vegas, Nevada 89154-4004 USA.

${ }^{4}$ Corresponding author.

\section{Introduction}

Recent years have seen the accumulation of a large body of evidence implicating the adult Drosophila mushroom bodies (MBs) in olfactory associative learning and memory: Single-gene mutants selected for gross defects in adult MB structure exhibit corresponding defects in olfactory associative learning (Heisenberg et al. 1985; Heisenberg 1989; Brotz et al. 1996; de Belle and Heisenberg 1996). Mutations in several genes expressed predominantly in the MBs cause defective olfactory learning: for example dunce(dnc), rutabaga (rut), DC0, and Pka-R1 (Nighorn et al. 1991; Han et al. 1992; Skoulakis et al. 1993; Goodwin et al. 1997), which encode elements of the CAMP signaling cascade implicated in memory formation across a range of animal phyla (Hawkins et al. 1993); volado a member of the $\alpha$-integrin gene family (Grotew iel et al. 1998); and leonardo a member of the 14.3.3 gene family (Skoulakis and Davis, 1996). Associative odor learning is virtually abolished in flies in which the MBs have been selectively ablated by hydroxyurea (HU) treatment during a critical window of larval development (de Belle and Heisenberg 1994). Finally, specific disruption of G-protein coupled pathways in the MBs causes extreme learning defects (Connolly et al. 1996).

Olfactory associative learning is not an adultspecific trait, how ever. Drosophila are capable of olfactory learning as larvae (Aceves-Pina and Quinn 1979), and appear able to recall their training as adults (Manning 1967; Jellies 1981; Cambiazo et al. 1994). Assuming that the latter ability conforms to current synaptic models for basic learning pro-

LEARN IN G \& MEM ORY 5:102-114 @ 1998 by Cold Spring Harbor Laboratory Press ISSN 1072-0502/98 \$5.00

$$
\begin{array}{lllllllllllllllll}
\hline & E & A & R & N & I & N & G & \underset{102}{\mathbf{X}} & M & E & M & O & R & Y
\end{array}
$$


cesses (Haw kins et al. 1993), one might expect the neuropil(s) responsible for memory retention to persist, as do the MBs (see below) through the otherwise large-scale neuronal reorganization that takes place during metamorphosis.

The adult Drosophila MBs are large paired neuropils emanating from cell bodies in the caudodorsal protocerebrum (Power 1943). They comprise some 2000-3000 intrinsic neurons, Kenyon cells (KCs), the precise number depending on a range of genetic and environmental factors (Technau and Heisenberg 1982; Technau 1984; Heisenberg et al. 1995). KC cell bodies each send a single neurite into a region of dendritic arborization known as the calyx. The latter is innervated largely by efferents from the paired antennal lobes (ALs), the first relay station for chemosensory processing (Stocker 1994). KC axons project rostrally from beneath the calyx as a densely packed and stalk-like structure known as the pedunculus. At the frontal margin of the brain, the latter gives rise to a number of projections: a small lateral spur (or knee); one dorsally projecting lobe $(\alpha)$; and two medially projecting lobes ( $\beta$ and $\gamma$ ) (see Yang et al. 1995). As we show here, there is an additional dorsally projecting lobe, which we call $\delta$. A summary diagram of the adult Drosophila MBs is show $n$ in Figure 1.

MBs, unlike most other elements of the adult brain, exhibit developmental continuity. The KCs arise from just four mushroom body neuroblasts (MBNbs) per hemisphere (Ito and Hotta 1992). These begin proliferating in the embryo and are continually active until late pupal stage. In addition to $K C s$, the four MBNbs also give rise to a small population of glia in the KC cortex (Ito et al. 1997). Rudimentary MBs, topologically similar to the adult MBs, first become apparent betw een stages 14 and 17 of embryonic development (Tettamanti et al. 1997). They continue to grow by the addition of newly born KCs throughout larval development. During metamorphosis, much of the larval brain is remodeled via a process of neural degeneration, birth, and regrowth. In the case of the MBs, KC fiber number drops sharply during the first few hours of metamorphosis, but then rises again within $24 \mathrm{hr}$. KC body number appears to increase steadily throughout the same period (Technau and Heisenberg 1982). These observations were taken to imply massive degeneration of larval $K C$ axons, and regrowth of imaginal fibers from existing $\mathrm{KC}$ bodies. Approximately 500 relatively narrow KCs forming a core element of the developing pedun-

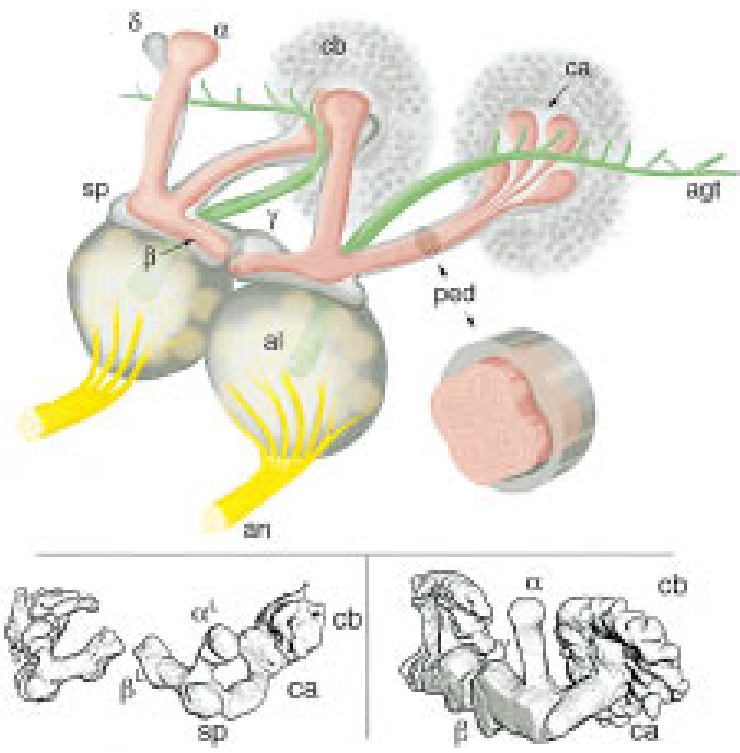

Figure 1: Drosophila mushroom bodies. (Top) Schematic representation of the adult D rosophila olfactory system. Olfactory input from sensory receptors on the antennae reaches the antennal lobes (al) via the antennal nerve (an). The glomerular arrangement of the AL is indicated. The $A L$ also receives olfactory input from the maxillary palps (not shown). Among the outputs of the $\mathrm{AL}$ is the antennal glomerular tract (agt), projecting caudally toward the MB calyx (ca), and then to the lateral protocerebrum. The calyx contains the dendritic arbors of $\mathrm{KCS}$ and is half contained within a rind of $\mathrm{KC}$ cell bodies (cb). KC axons arising within the calyx project rostrally via a stalk-like structure known as the pedunculus (ped). Upon reaching the rostral margin of the central brain, the pedunculus gives rise to a spur-shaped lateral projection (sp), and to four lobes $(\alpha, \beta, \gamma, \delta)$. $\beta$ and $\gamma$ project toward the midline, where they almost abut. $\alpha$ and $\delta$ project to the dorsal margin of the brain. MBs exhibit a clear and continuous fourfold symmetry within the cell body layer (four adjacent "clouds"), the calyx and the pedunculus (four internal tracts, each of which has a concentric organization). As they transit the pedunculus the four tracts merge, and it is their projections that form the $\alpha$ and $\beta$ lobes. Surrounding the four peduncular tracts is a sheath of KC fibers projecting via the spur to form the $\gamma$ lobe and at least some elements of the $\delta$ lobe. The inset is a schematic representation of a crosssection though the pedunculus, illustrating its internal organization. (Bottom) Surface-rendered models of late third instar larval (left) and adult (right) M Bs as visualized in $P\{G A L 4\}$ line $201 Y$. The main MB structures are indicated, with the exception of the adult $\delta$ lobe, which is not encompassed by the expression pattern of line 201Y.

culus were interpreted as a nondegenerating subset, possibly acting as a guide for new ly extending $\mathrm{KC}$ axons. 
$\mathrm{KCs}$ of the adult MBs fall into at least three broad classes ( $Y$ ang et al. 1995). One class is that of KCs projecting to the $\alpha$ and $\beta$ lobes alone, and which exhibit longitudinal fourfold symmetry within the cell body layer (four clusters), the calyx (four quadrants), and the pedunculus (four tracts). Each of the four peduncular tracts is constructed from a number of concentric elements, apparently continuous with concentric "shells" of the $\alpha$ and $\beta$ lobes. A second class is that of KCs projecting to the $\gamma$ lobe alone. Although these KCs must arise from the same four neuroblasts as do the $\alpha / \beta$ lobe $K C s$, they exhibit no obvious fourfold symmetry. Rather, cell bodies and dendrites of this class appear evenly distributed across the cell body layer and within the calyx. Within the pedunculus, KC axons of this class form a sheath that surrounds the four tracts described above. They subsequently project to the $\gamma$ lobe via the spur, a route not taken by $\alpha$ and $\beta$ fibers. Some KC axons appear to project to both the $\gamma$ and $\delta$ lobes, and thus constitute a third class, distinct from that which projects to the $\gamma$ lobe alone.

The MB subdivisions described above were largely identified using the $P\{G A L 4\}$ enhancer-trap system, which has provided an unprecedented set of cell-type-specific neural markers ( $Y$ ang et al. 1995). Here we follow the development of various $\mathrm{P}\{\mathrm{GAL} 4\}$ expression patterns at timed intervals through metamorphosis. Furthermore, we have used the $\mathrm{HU}$ ablation technique to assess the contribution of prelarval MB elements to the larval and adult structures.

Combined, these approaches have allowed us to address the following: Does each of the four MBNbs contribute to all adult lobes, or do the different lobes arise from different neuroblasts? If any of the embryonic KCs survive to adulthood, we would expect to find them in whichever of the MB structures forms earliest during development. Is this the $\gamma$-lobe, as previously hypothesized (Yang et al. 1995)? What are the structural features of the larval MBs and how do these relate to the adult structures? Some, but not all, KC axons have been show $\mathrm{n}$ to degenerate during early metamorphosis (Technau and Heisenberg 1982). Is this degeneration widespread or is it restricted to specific MB substructures.

In addition to $P\{G A L 4\}$ lines marking the $K C S$, we have lines that mark putative AGT (Fig. 1; MB input) and $\gamma$ lobe exit (MB output) neurons. The effects of $\mathrm{HU}$ ablation on their projection patterns is described.

\section{Materials and Methods}

\section{DROSOPHILA}

The lines described here were isolated from a screen of 1800 independent $P\{G A L 4\}$ lines for patterns of expression in adult brain (Yang et al. 1995; Table 1). The $\cup_{A S}$-lacZ secondary reporter line, homozygous for an insertion on the second chromosome, was provided by Andrea Brand (Brand and Perrimon 1993). Flies were maintained on standard corn meal/yeast/agar medium at $25 \pm 1{ }^{\circ} \mathrm{C}$ and $40 \%$ relative humidity with a $12-\mathrm{hr}$ light/ 12-hr dark regime.

\section{COLLECTION OF PUPAE}

Newly immobile prepupae were collected at 30-min intervals, sexed, and timed. They were then reared singly under the same conditions as normal pupae until ready for dissection. Both male and female pupae were dissected at 4-hr intervals (sometimes $2 \mathrm{hr}$ ). Dissection times were accurate to $\pm 5 \mathrm{~min}$ and collection times to $\pm 30 \mathrm{~min}$.

\section{HU ABLATION}

In the newly hatched larva, most neuroblasts are dormant with the exception of the four MBN bs and a single other that contributes to the AL (de Belle and Heisenberg 1994; Prokop and Technau 1994; Stocker et al. 1997).

HU ablation was performed according to de Belle and Heisenberg (1994). New ly hatched larvae (30 min \pm 30 min after larval hatching) were fed a 4-hr pulse of heat-killed yeast suspension containing $\mathrm{HU}$ at either 10 or $50 \mathrm{mg} / \mathrm{ml}$ concentration. Control animals were fed yeast only. Larvae were then transferred to standard medium without HU. The success of ablation was determined by examination of autofluorescent brain sections from representative individuals. No significant difference between using $\mathrm{HU}$ at either 10 or $50 \mathrm{mg} / \mathrm{ml}$ concentration was observed.

A minimum of 10 individual brains was examined by confocal microscopy for each ablated $P\{G A L 4\}$ expression pattern. With the exception of $X$-linked insertions, both male and female brains w ere examined, but no differences w ere observed.

\section{IMMUNOHISTOCHEMISTRY}

Intact adult brains were dissected under phosphate-buffered saline (PBS), fixed in $4 \%$ paraform- 
Table 1: HU ablation

\begin{tabular}{|c|c|c|c|c|c|c|c|c|c|c|}
\hline \multirow{2}{*}{$\begin{array}{l}P\{G A L 4\} \\
\text { line }\end{array}$} & \multirow{2}{*}{$\begin{array}{l}\text { Insertion } \\
\text { site }\end{array}$} & \multicolumn{8}{|c|}{ Relevant adult expression pattern } & \multirow[b]{2}{*}{ Reference } \\
\hline & & AN & $A L$ & $A G T$ & $\alpha$ & $\beta$ & $\gamma$ & $\delta$ & $\gamma$-exit & \\
\hline $30 Y$ & $70 \mathrm{E}$ & & & & - & - & + & - & & Yang et al. (1995) \\
\hline $43 Y$ & $2 \mathrm{C}$ & & & & - & - & & & + & this study \\
\hline $201 Y$ & $56 \mathrm{D}$ & & & & - & - & + & & & Yang et al. (1995) \\
\hline $238 Y$ & $48 \mathrm{C}$ & & & & - & - & - & - & + & Yang et al. (1995) \\
\hline c35 & $44 \mathrm{~A}$ & & - & & - & - & - & - & & Yang et al. (1995) \\
\hline c133 & 11D & + & & + & & & & & & this study \\
\hline$c 492 b$ & $49 C$ & & - & & - & - & - & - & & this study \\
\hline c739 & $40 \mathrm{~A}$ & & - & & - & - & & & & Yang et al. (1995) \\
\hline c742 & $8 \mathrm{C}$ & + & & & & & & & + & this study \\
\hline c772 & $42 \mathrm{~A}$ & & - & & - & - & + & - & & Yang et al. (1995) \\
\hline
\end{tabular}

Summary of the effect of $\mathrm{HU}$ ablation on the lines examined. The neuropil regions are as follow s: (AN) antennal afferents via antennal nerve; (AL) antennal lobe intrinsic neurons; (AGT) neurons in the antennoglomerular tract; $(\alpha)$ KC axons in the $\alpha$ lobe; $(\beta)$ KC axons in the $\beta$ lobe; $(\gamma)$ KC axons in the $\gamma$ lobe; $(\delta)$ KC axons in the $\delta$ lobe; ( $\gamma$-exit) M B extrinsic neurons connecting with the $\gamma$ lobe. $(+)$ Some (though not necessarily all) of the axons are present after HU treatment; $(-)$ axons in which staining is lost after $\mathrm{HU}$ treatment.

aldehyde for $30 \mathrm{~min}$, and washed twice for $1 \mathrm{hr}$ in PAT (PBS containing $1 \%$ Sigma cold fraction $\checkmark$ bovine serum albumin and $1 \%$ Triton X-100). They were incubated overnight in $3 \%$ normal goat serum (Scottish Antibody Production Unit) containing rabbit polyclonal anti- $\beta$-gal antibody (Cappel) diluted 1:2000 in PAT; washed three times in PAT for $1 \mathrm{hr}$; incubated overnight with secondary antibody (fluorescein-labelled goat anti-rabbit IgG; Vector Labs) diluted 1:250 in PAT; washed twice for $1 \mathrm{hr}$ in PAT, and once for $5 \mathrm{~min}$ in PBS. All of the above was carried out at room temperature. Stained brains were mounted in VectaShield (Vector).

\section{CONFOCAL MICROSCOPY, 3D RECONSTRUCTION, AND IMAGE PROCESSING}

Whole-mount stained brains were examined with a Molecular Dynamics Multiprobe laser scanning confocal microscope. The excitation (480 $\mathrm{nm})$ and emission $(530 \pm 15 \mathrm{~nm})$ barrier filters used were appropriate to the fluorescein-based label of the secondary antibody. Either a single brain hemisphere or the central brain region alone was scanned. Three dimensional reconstructions (maximum intensity) were performed using the program ImageSpace 3.1 (Molecular Dynamics).

\section{Results}

\section{HU ABLATION OF MBS}

Ten independent $\mathrm{P}\{\mathrm{GAL} 4\}$ lines were subjected to HU treatment (Table 1). Adult brains were subsequently dissected, stained, and examined. Both by autofluorescence (not shown) and by confocal microscopy (Figs. 2-5), non-MB neuropil, with the exception of the ALs, appears unaffected by HU treatment. This further confirms that $\mathrm{HU}$ treatment affects only neuroblasts giving rise to the MBs and ALs.

Eight of the HU-treated P $\{G A L 4\}$ lines normally show GAL4-dependent $\beta$-gal expression in the adult MBs, though in different subpopulations of $\mathrm{KCs}$. For five of these lines, three of which are illustrated in Fig. 2, HU treatment causes complete ablation of the adult KC staining pattern (see Table $1)$. For the three other lines, by contrast, we consistently observe $\mathrm{KC}$ fibers that survive ablation (Fig. 3), indicating an origin prior to larval hatching. In each case, the surviving fibers ( 50 or less per line) have medial projections that closely resemble the projection patterns of $\gamma$ lobe neurons (see Yang et al. 1995); no projections to the $\alpha, \beta$, or $\delta$ lobes were seen. In summary a subset of embryonic KCs, comprising a fraction of the normal $\gamma$ lobe, clearly survives to adulthood.

Of the 300 brains that we examined, 7 showed evidence of incomplete ablation (Fig. 4). Partially 

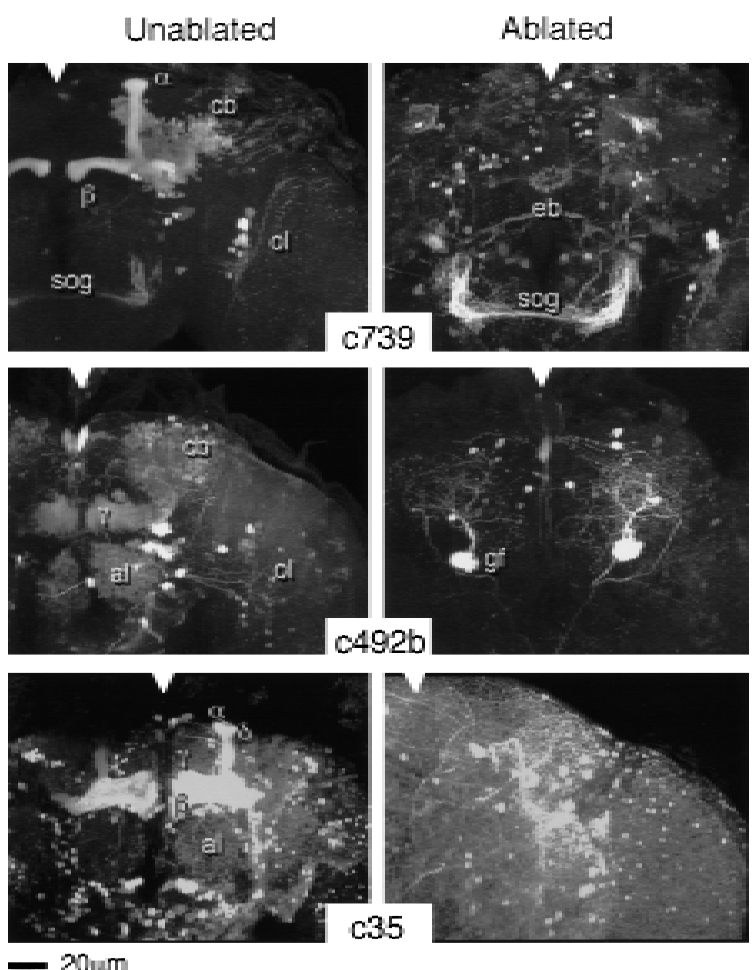

Figure 2: HU ablation of postembryonic KCs. Threedimensional reconstructions of adult brains from the indicated $P\{G A L 4\}$ lines. (Notches) Approximate position of the midline in each panel. (c739) The normal expression pattern reveals the $\alpha$ and $\beta$ lobes of the MBs, intrinsic neuronal components of the ALs (weak), the ellipsoid body-a component of the central complex (weak), together with elements of the optic lobes (ol) and the subesophageal ganglion (sog). (c492b) The normal pattern reveals large numbers of KCs within the $\alpha, \beta$, and $\gamma$ lobes ( $\alpha$ and $\beta$ less clear in this reconstruction), intrinsic neurons of the ALs (al), the giant fiber (gf, partly obscured by MB staining), and elements of the optic lobes. (c35) The normal pattern extends widely through central brain neuropil but is strongest within the MBs $(\alpha$, $\beta, \gamma$, and $\delta)$. In each case, the ablated pattern is selectively and completely depleted for $M B$ and $A L$ staining. Surrounding neuropil, as far as can be determined, appears normal.

ablated MB patterns in each case resembled the corresponding nonablated pattern with respect to lobe number and gross morphology. They were substantially thinner, however, and lacked evidence for fourfold symmetry at any level of organization. We infer that each of the observed partial ablation patterns represents the progeny of a single surviving $\mathrm{MBNb}$ and, thus, that the contribution of each MBNb to the four lobes is equivalent (see also Ito et al. 1997).

\section{ABLATION OF MB EXTRINSIC NEURONS}

Four of the $P\{G A L 4\}$ lines described in Table 1 exhibit reporter expression within MB extrinsic neurons. Line $\mathrm{c} 133$ reveals staining of what appear to be AGT fibers (see Fig. 1) connecting the ALs and the MB calyx (Fig. 5). HU treatment does not obviously affect the projection pattern of these neurons. How ever, HU treatment was shown to ablate

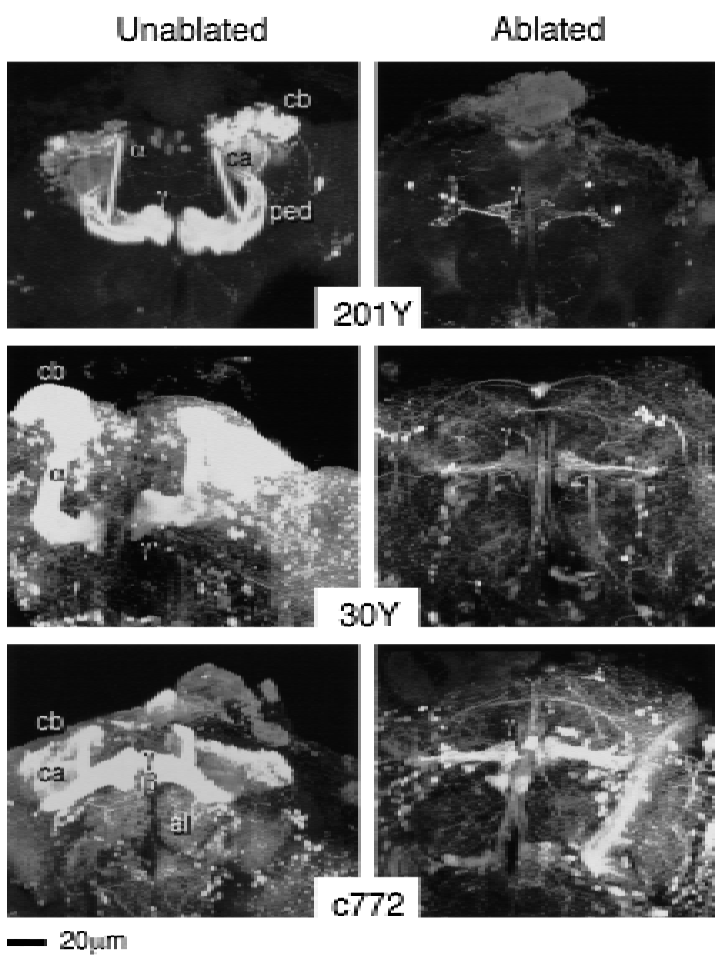

Figure 3: Survival of embryonic KCs. Three-dimensional reconstructions of adult brains from the indicated $P\{G A L 4\}$ lines. (201Y) The normal expression pattern reveals core elements of the $\alpha$ and $\beta$ lobes, $\gamma$ lobes, together with just a few other neurons. (30Y) Relatively extensive expression within the central brain includes strong MB staining (all four lobes). (c772) Again, relatively extensive expression within the central brain, strong MB staining (within all four lobes), and also a group of AL interneurons. In each case, the ablated pattern is selectively depleted for all MB staining except for a small group of $\mathrm{KCs}$, different numbers in each case, projecting towards the mid-line. After ablation, so few $\mathrm{KCS}$ remain relative to surrounding neuropil that threedimensional reconstructions of the entire brain tend to obscure them. The reconstructions shown are only of the lobe regions. In sections, however, surviving KCs can be followed from cell bodies of expected shape and size in the expected dorsoposterior location. They project to a very small calyx and send their axons through a narrow pedunculus. 

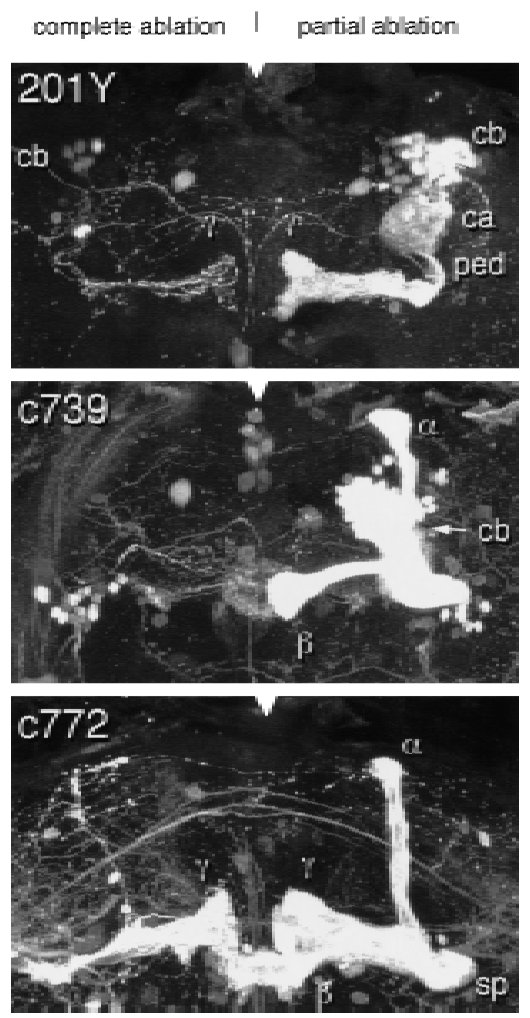

Figure 4: Partial ablation-unilateral. Three-dimensional reconstructions of partially ablated MBs from the indicated lines. All lobular elements of the normal adult pattern are represented in each case. The lobes are, however, much thinner that their nonablated counterparts (cf. Figs. 2,3). The $\alpha$ and $\beta$ lobes of $201 Y$ are not visible in this reconstruction but can be seen in the sections from which it was created. This is consistent with the results of a similar study by Ito et al. (1997).

other groups of AGT neurons in another study (Stocker et al. 1997).The other three lines, the clearest example of which is shown in Figure 5 (c742), have staining patterns corresponding to a group of fibres connecting the tip of the $\gamma$ lobe to a rostral area of the medial protocerebrum. Again, $\mathrm{HU}$ ablation has no obvious effect on their projection patterns. In particular, the bulbous shape corresponding to the tip of the normal $\gamma$ lobe is retained.

\section{ABLATION OF AL INTRINSIC AND EXTRINSIC NEURONS}

Three of the lines selected for HU treatment reveal staining corresponding to AL local interneurons, which have ramifications throughout the $A L$ (Figs. 2, lines c739 and C492b, and 3, line c772).
The three patterns differ with respect to the number of stained cell bodies, implying different subsets of such local interneurons. All are eliminated by $\mathrm{HU}$ treatment.

Two other lines reveal expression in groups of $A L$ afferents that enter the $A L$ via the antennal nerve, and that arborize within different subsets of $A L$ glomeruli (Fig. 5). Bar an overall reduction of $A L$ size, HU treatment had no obvious effect on the projection patterns of these neurons, or on the morphology of the glomeruli.

\section{LARVAL MBS}

Among $P\{G A L 4\}$ lines that reveal the adult MBs, approximately half also reveal the larval MBs (Tettamanti et al. 1997). Examples of expression patterns in the third instar larval MB are show $n$ in the first column of Figure 6 . The larval MB is topologically similar to that of the adult (see also Fig. 1), comprising a cortical array of cell bodies, a calyx, a pedunculus and a lobe system. Closer examination of the larval MB reveals some striking differences from the adult structure, how ever.

The most obvious difference is with respect to the number of lobes. The larval MB appears to have just two lobes; one projecting dorsally and one projecting medially (see Figs. 1 and 6). Both lobes, and in particular the medially projecting lobe, have bulbous out-sw ellings at their tips (c.f. the adult $\gamma$ lobe; Yang et al. 1995). Also, unlike the adult structure, the larval pedunculus makes a turn towards the midline before giving rise to the lobes. Close to the point of bifurcation there is a small lateral projection that may be analogous to the adult spur.

Internally, the larval pedunculus appears to have a simple concentric organization; each pattern reveals one or more sheaths of fibers surrounding an unstained core (Fig. 7). Unlike the adult pedunculus, we find no evidence of a fourfold symmetric internal tract system, although we sometimes observe two tracts exiting the calyx to join the pedunculus (e.g., line 30Y, Fig. 7). We do see evidence for four loose clusters of cell bodies, however, each of which is presumably derived from a single MBNb (e.g., line 30Y, Fig. 7).

Leaving aside for the moment the question of developmental continuity, the clearest example of the difference between the larval and adult MBs is provided by line 201Y (Figs. 1 and 6). This line exhibits reporter expression in the adult $\gamma$ lobe and in core elements of the adult $\alpha$ and $\beta$ lobes. 
Armstrong et al.

Figure 5: Ablation of $M B$ and $A L$ extrinsic neurons. Three-dimensional reconstructions of adult brains from the indicated $P\{G A L 4\}$ lines. (c133) The normal expression pattern (left) reveals two relevant neuronal types. O ne enters the $A L$ via the $A N$, and terminates ipsi- and contralaterally within a set of dorsomedial glomeruli. The second appears to correspond to a subset of AGT fibers. These project ipsi- and contralaterally to the calyx (ca) and to the lateral protocerebrum/lateral horn (Ih). The ablated pattern (right) is remarkably similar, except for a reduction in volume of the AL. (c742) The normal expression pattern (left) reveals two relevant neuronal types. $O$ ne enters the $A L$ via the AN, terminating ipsi- and contralaterally within a subset of glomeruli. The second appears to correspond to $\gamma$-exit fibers. Each hemisphere contains a group of cell bodies dorsomedial to the M B lobes that projects to a region identical in shape and position to the tip
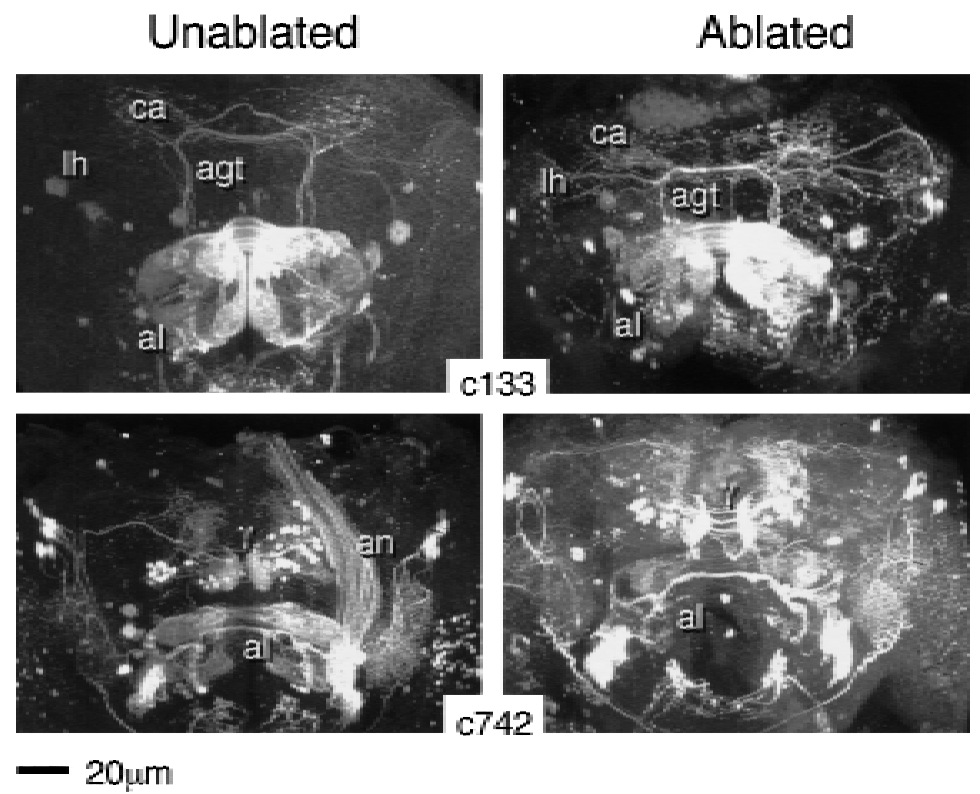

of the $y$ lobe. Projections to the medial protocerebrum, and via a few longer fibers to the lateral protocerebrum, are revealed. Additional and unrelated staining occurs within the lateral protocerebrum and the optic lobes. The ablated pattern (right) is remarkably similar, except for a reduction in AL volume.

The latter are continuous with four tracts internal to the pedunculus, whereas the former is continuous with a sheath of fibers that surrounds them (Yang et al. 1995). In the larval MB, in contrast, $201 Y$ reveals outer shells of both larval lobes, continuous with an outer shell of the pedunculus (Fig. 7).

Given that the larval and adult lobes appear to have rather different internal structures, we consider it inadvisable to use the same terminology to describe them. For this reason, we will use the terms $\alpha^{\mathrm{L}}$ and $\beta^{\mathrm{L}}$ when referring to the larval lobes.

\section{DEGENERATION OF THE LARVAL MBS}

$\mathrm{KC}$ expression patterns were follow ed at 2- to 4-hr intervals during the first $48 \mathrm{hr}$ of postlarval development, starting with newly immobile prepupae. No obvious changes were seen for at least a further $8 \mathrm{hr}$, that is, until $\sim 2 \mathrm{hr}$ after puparium formation (APF). Between that time and $16 \mathrm{hr}$ APF, all of the patterns undergo change, though to different extents (Fig. 6, second column). This is seen at its most extreme in $\alpha^{L}$, in which reporter expression appears to fragment and then to disappear completely (Fig. 6 , third column) in lines $201 Y$ and 203Y. Changes of $\alpha^{L}$ in other lines, although less dramatic, always appear to be accompanied by a reduction in diameter.
The apparent degeneration of at least some elements of $\alpha^{\mathrm{L}}$ is accompanied by similar changes within $\beta^{\mathrm{L}}$, though for this lobe at least some staining persists in all of the lines examined, most extensively in lines that are the least affected with respect to $\alpha^{L}$. Staining intensity in the pedunculus was also reduced, with respect to that of the cell body layer, in all of the patterns that we follow ed.

\section{CONSTRUCTION OF ADULT MBS}

Structures clearly recognizable as, and developmentally continuous with, the adult MB lobes appear in most lines from $\sim 26 \mathrm{hr}$ APF. Earliest to appear is the medially projecting $\gamma$ lobe, which appears to grow out of the remnant of $\beta^{\mathrm{L}}$. Simultaneous appearance of $\alpha$ and $\beta$ lobes occurs somewhat later, with a timing that varies from line to line. Their earliest appearance is in lines 30Y, $203 Y$, and 238Y, in which narrow $\alpha$ and $\beta$ lobes become visible almost as soon as the $\gamma$ lobe becomes recognizable. In line 203Y, for which the adult $\alpha$ and $\beta$ lobes have characteristic unstained cores, the newly formed $\alpha$ and $\beta$ lobes exhibit staining throughout. The unstained cores only become apparent later than $36 \mathrm{hr}$ APF. This is around the same time that staining of $\alpha$ and $\beta$ lobe cores appears in line 201Y. By $40 \mathrm{hr}$ APF, the $\alpha, \beta$, and $\gamma$ lobes look very similar to those of the adult. 


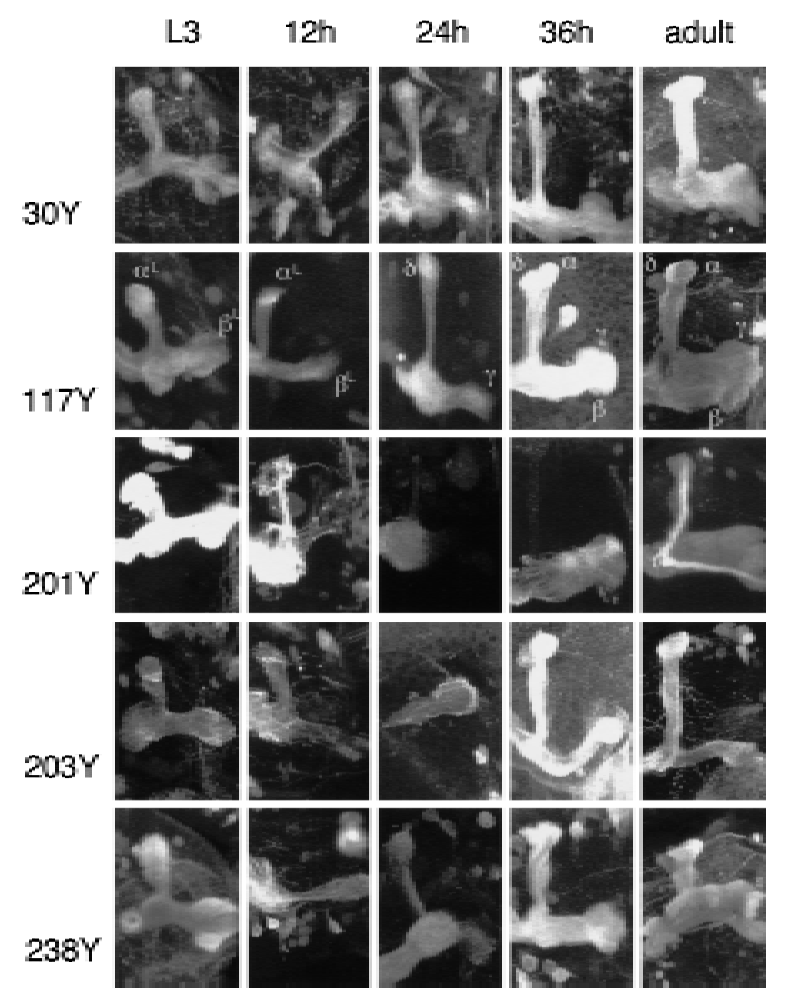

Figure 6: $\quad$ Timed study of $P\{G A L 4\}$ expression patterns. Three-dimensional reconstructions of lobe regions from the indicated $P\{G A L 4\}$ lines (see also Table 2). Larval and adult patterns are in the leftmost and rightmost columns, respectively. Three representative pupal stages are shown for each line. The second column corresponds to early pupal development ( 12 hr APF), the third column to midpupal development ( $24 \mathrm{hr}$ APF), and the fourth column to later pupal development $(\sim 36$ hr APF).

Lying adjacent to, and to an extent coiling around the adult $\alpha$ lobe we can discern a second dorsally projecting structure. This lobe, which we call $\delta$, may be developmentally continuous w ith $\alpha^{\mathrm{L}}$, and appears at the same time as the $\gamma$ lobe. The relationship between $\alpha$ and $\delta$ is most clearly seen in lines 117Y and 238Y (Fig. 6). Discrete $\alpha$ and $\delta$ lobes persist to adulthood.

\section{LARVAL ORIGIN OF THE ADULT $\gamma$ LOBE}

In all of the lines that we have examined, with only a single exception (line c739), staining in the larval MB lobes correlates with staining of the $\gamma$ lobe in the adult MB. Furthermore, lines that stain most extensively in the $\gamma$ lobes show the least degree of degradation during the initial phase of metamorphosis (Fig. 6, lines 30Y, 117Y, and 238Y).
The expression pattern of line 201Y contains $\mathrm{KCs}$ that survive HU treatment (Fig. 3), and that form an adult "lobe" with a $\gamma$-like structure. Surviving fibers can also be seen at the third larval instar stage (Fig. 8), in which they project to both of the larval lobes. This implies developmental continuity between the larval lobes and the adult $\gamma$ lobe, rather than the adult $\alpha$ and $\beta$ lobes. In the unablated larval brain, $201 Y$ staining (which must include the HU-resistant subset) is restricted to an outer shell of the pedunculus (Fig. 5). It thus appears, as we have hypothesized previously (Yang et al. 1995), that the MB grow s from the inside out, such that the earliest fibers come to reside on the outside.

\section{Discussion}

\section{ADULT KCS OF EMBRYONIC ORIGIN}

In newly hatched first instar larvae at the time of $\mathrm{HU}$ treatment, the four MBNbs and a single lateral neuroblast $(\mathrm{LNb})$ are the only proliferating
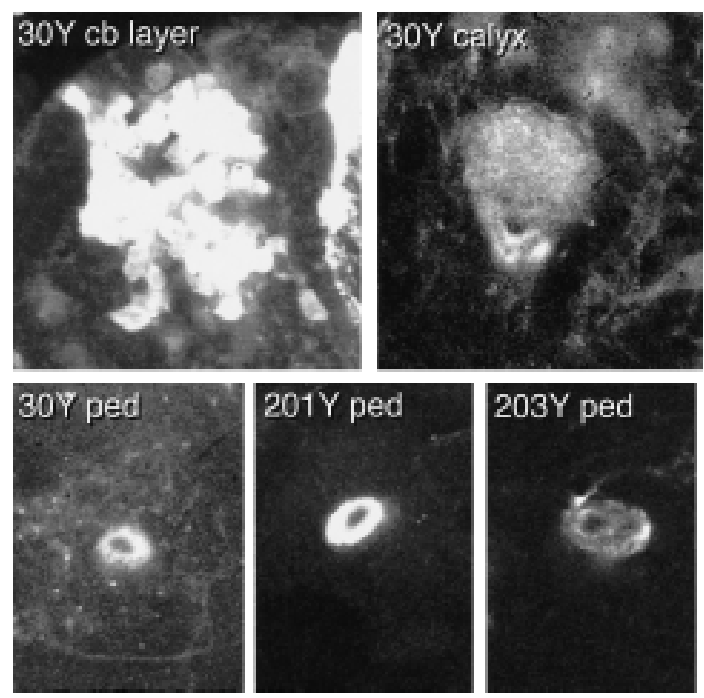

Figure 7: The late larval MBs. Selected confocal sections through the late third instar larval cell body layer (top left) and calyx (top right) of line 30Y, and through the pedunculus of $30 \mathrm{Y}$ and two other lines (bottom). The cell body region of $30 \mathrm{Y}$ appears as four clusters, each surrounding an unstained core (presumably containing the corresponding MBNbs). The junction between the calyx and the pedunculus in line $30 \mathrm{Y}$ appears as two tracts with unstained cores. These are continuous, with the stained and unstained components of the $30 \mathrm{Y}$ pedunculus. Staining of the larval pedunculus (bottom panels) appears as one or more concentric rings surrounding an unstained core. 
Armstrong et al.

Figure 8: Effect of $\mathrm{HU}$ ablation on the larval MBs. Three-dimensional reconstruction of the MB pattern in $\mathrm{HU}$-treated late third larval instar 201Y. The ablated larval MB shows all the features of the nonablated larval pattern, albeit with much narrower lobes.

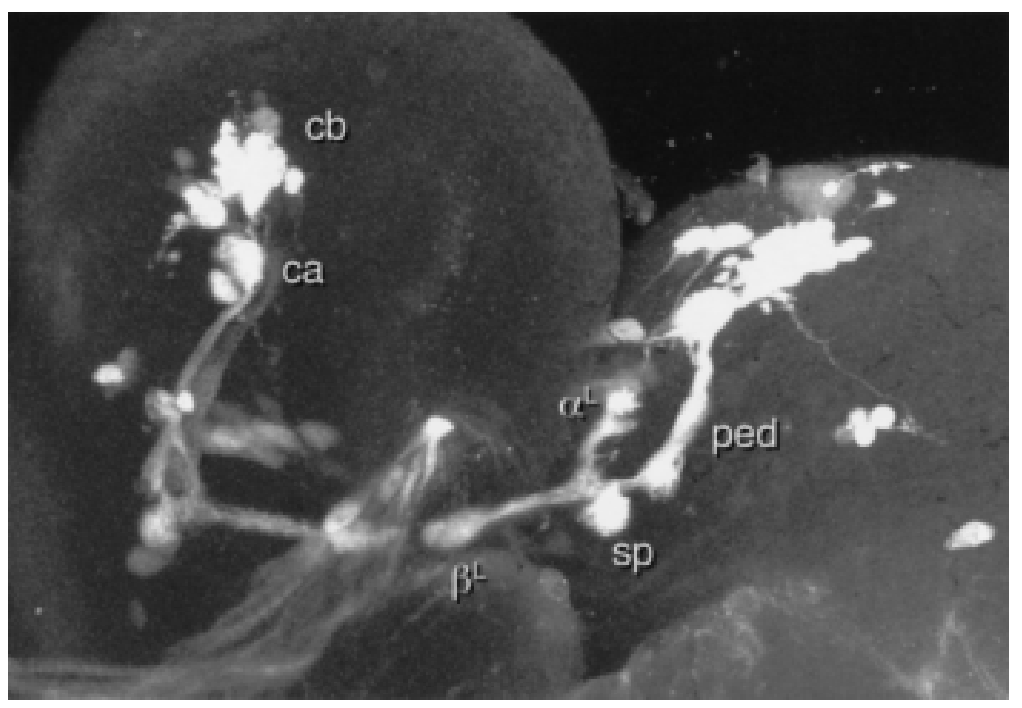

neuroblasts in each brain hemisphere (Truman and Bate 1988; Prokop and Technau 1991; Ito and Hotta 1992). That HU ablates MB intrinsic fibers (KCS) together with a group of $\mathrm{AL}$ neurons (Stocker et al. 1997) conforms to this pattern of neuroblast activity.

Here we describe the effects of larval HU ablation on eight P[GAL4] lines with expression patterns restricted to different subsets of the adult $\mathrm{KCs}$. In the case of five lines, not a single marked $\mathrm{KC}$ was apparent in the adult brain. In three lines, how ever, staining attributable to a small number of $K C s$, consistent between individuals of the same line, was clearly recognizable. Two possibilities for their origin exist. They are either formed prior to HU treatment, or they arise from neuroblasts active at a later stage of development. Despite careful BrdU incorporation studies, no additional MB neuroblasts have been found (Ito and Hotta 1992). We thus conclude that at least some embryonic KCs survive to adulthood.

The three surviving patterns are clearly different from one another, at least in terms of $\mathrm{KC}$ number (Fig. 3). What they have in common are axonal trajectories closely resembling those of normal adult $\gamma$ lobe fibers (Yang et al. 1995), and they occur only in lines that normally exhibit $\gamma$ lobe staining. Surviving KCs contribute to merely a portion of the $\gamma$ lobe, however, implying that some (probably most) $\gamma$-lobe neurons are born after larval hatching. We see no evidence of $\alpha$ or $\beta$ lobe neurons surviving after complete ablation in any line. Nor do we observe axons projecting to the $\delta$ lobe. These results are consistent with our previous hypothesis that $\mathrm{KCs}$ within the adult $\gamma$ lobe are the earliest MB elements to arise during development (Yang et al. 1995).

The number of $\mathrm{KCs}$ at hatching has been previously estimated as $\sim 300$ (Technau and Heisenberg 1982). Here, we observe little more than 100 adult KCs in the three surviving patterns combined. Whereas the lines we chose to examine encompass large numbers of $\mathrm{KCS}$, in combination representing a substantial proportion of the total KC count, they may just fail to include expression patterns corresponding to the unaccounted 200 fibers. Alternatively, some of the embryonic fibers may be lost during larval and pupal development.

\section{RELATIONSHIP BETWEEN THE FOUR MBNbS}

Adult MBs exhibit clear fourfold symmetry (Yang et al. 1995). Fibers that project to the $\alpha$ and $\beta$ lobes emanate from four clusters of cell bodies; occupy four quadrants of the calyx; and are contained within four tracts internal to the pedunculus (Fig. 1). In contrast, KCs associated with the $\gamma$ lobes appear to be diffusely scattered across the cell-body region (Fig. 1; Yang et al. 1995); they reveal the entire calyx volume; and they form a sheath in the pedunculus, not obviously subdivided, that surrounds the four internal tracts. We have previously hypothesized a causal relationship between the fourfold symmetry of the adult Drosophila MBs and an origin from four equivalent neuroblast precursors (Yang et al. 1995) Alternatively, each neuroblast might give rise to a specific class of Kenyon cell (e.g., each MBNb specific for a particular lobe).

\section{$\begin{array}{lllllllllllllll}L & E & A & R & N & \text { I } & N & G & \underset{\mathbf{1 1 0}}{\mathbf{X}} & M & E & M & O & R & Y\end{array}$}


MUSHROOM BODY DEVELOPMENT

Evidence presented here, and in another study (Ito et al. 1997), favors the former interpretation. In each of several partially ablated patterns we find a much thinner than usual MB comprising a single cell-body cluster; an undivided calyx; an undivided pedunculus; and all of the normal lobes. Thus each of the four cell-body clusters, calyx units, and peduncular tracts would appear to arise from separate, and largely if not entirely equivalent, neuroblasts. Each MBNb also contributes a proportion of $\gamma$-lobe KCs, both before and after hatching. The four MBNbs would thus appear largely equivalent with respect to their contributions to the adult MB (see also Ito et al. 1997). The four MBNbs are not entirely equivalent in terms of patterns of gene expression (and quite possibly function), however; one P\{GAL4\} line has been described that reveals only two MBNbs and their progeny at a very early stage of MB development (Tettamanti et al. 1997).

\section{LARVAL MBS}

Use of the $P\{G A L 4\}$ system has allowed us to describe the detailed structure of the late larval MBs for the first time. Although topologically similar to the adult MBs, they have some distinct features. In particular, we see only two lobes ( $\alpha^{\mathrm{L}}$ and $\beta^{L}$ ) compared with the four lobes observed in the adult. There is also a simple concentric organization of the pedunculus rather than the fourfold symmetrical arrangement of the adult. In terms of lobe morphology, the two larval lobes appear most like the adult $\gamma$ lobe, having bulbous out-sw ellings at their tips. In addition to the two lobes, the larval MB exhibits a spur-like structure; a spur, continuous with the $\gamma$ lobe, is also seen in the adult MB (Yang et al. 1995). Despite absence of fourfold symmetry in the pedunculus, the larval KC cell bodies are clearly arranged as four clusters, each presumably arising from, and surrounding, one of the four MBNbs.

Although the core of the larval pedunculus is known to contain KCs (Technau and Heisenberg 1982), we have yet to find a line that reveals it. Because we selected our lines on the basis of adult MB expression, it may be that the core fibers are a larval-specific feature with their ow $n$ gene expression characteristics. Alternatively, we may just not have looked at enough lines, or the core fibers may be too young to have accumulated sufficient $\beta$-gal reporter.

\section{MB METAMORPHOSIS}

Significant changes in MB expression patterns are observed during metamorphosis. In the case of several lines (see Table 2) the changes were so sudden and dramatic that they could only be reasonably attributed to abrupt changes in enhancer activity (Armstrong and Kaiser 1997). For the purposes of this study we describe only lines in which we observe gradual changes in their expression patterns. Taken together, they present a relatively consistent developmental story.

Puparium formation is associated with a significant reduction in the number of $\mathrm{KC}$ profiles seen in cross-sections of the pedunculus (Technau and Heisenberg 1982). Having dropped by almost $50 \%$ by $12 \mathrm{hr}$ APF, the number of profiles then climbs again, reaching a plateau at $\sim 48 \mathrm{hr}$ APF. Here we describe a temporally correlated reorganization of the MB lobes, first involving degeneration of the two larval lobes, to an extent that varies from line to line, and then a gradual appearance of the adult structures. Lines that show the least evidence of degeneration during early metamorphosis are those that stain all four lobes of the adult MBs.

In light of the changes in $\mathrm{KC}$ fiber number described by Technau and Heisenberg (1982), degeneration of elements of the larval lobes is most easily interpreted as degeneration of the relevant $\mathrm{KC}$ axons. The extent and precise nature of the degeneration is difficult to judge precisely, however. It is possible that all KCs participate, and that "nondegenerating elements" of the staining patterns represent an equilibrium betw een degeneration and outgrowth. We suspect, however, that persistent elements of lobular staining reflect persistence of at least a proportion of larval KC axons through to adulthood.

Our HU ablation studies imply that axons of the earliest KCs come to reside in outer shells of both the larval and the adult pedunculus. This is consistent with growth of the MBs from the inside out, such that the core of the pedunculus, at any particular developmental stage, will contain the youngest $\mathrm{KC}$ fibers. Fibers at the core of the developing pedunculus exhibit particularly thin axonal profiles (Technau and Heisenberg 1982).

\section{ORIGIN OF THE ADULT LOBES}

A recognizable $\gamma$ lobe first appears at $\sim 24-32$ hr APF (Fig. 6; middle column). It appears to grow out from the remains of $\beta^{\mathrm{L}}$, and is "complete" after 
Armstrong et al.

Table 2: Pupal development of $P\{G A L 4\}$ patterns

\begin{tabular}{|c|c|c|c|c|c|c|c|c|c|c|c|c|c|c|c|c|c|}
\hline \multirow[b]{3}{*}{ Line } & \multirow[b]{3}{*}{ Site } & \multicolumn{15}{|c|}{ Developmental expression } & \multirow[b]{3}{*}{ Reference } \\
\hline & & \multirow{2}{*}{$\begin{array}{l}\mathrm{L3} \\
\mathrm{MB}\end{array}$} & \multicolumn{2}{|c|}{ early pupal } & \multicolumn{4}{|c|}{ mid-pupal } & \multicolumn{4}{|c|}{ late pupal } & \multicolumn{4}{|c|}{ adult } & \\
\hline & & & $\alpha^{\mathrm{L}}$ & $\beta^{\mathrm{L}}$ & $\alpha$ & $\beta$ & $\gamma$ & $\delta$ & $\alpha$ & $\beta$ & $\gamma$ & $\delta$ & $\alpha$ & $\beta$ & $\gamma$ & $\delta$ & \\
\hline $30 Y$ & $70 \mathrm{E}$ & ++ & ++ & ++ & + & ++ & ++ & ++ & ++ & ++ & ++ & ++ & ++ & ++ & ++ & ++ & $\begin{array}{c}\text { Yang et al. } \\
\text { (1995) }\end{array}$ \\
\hline $82 Y$ & $? ?$ & & & & + & + & & & ++ & ++ & + & & ++ & ++ & + & & this study \\
\hline $103 Y$ & $2 \mathrm{D}$ & & & & & & & & ++ & ++ & ++ & ++ & ++ & ++ & ++ & ++ & $\begin{array}{c}\text { Tettamanti et } \\
\text { al. (1997) }\end{array}$ \\
\hline $117 Y$ & $34 C$ & ++ & + & ++ & + & + & ++ & ++ & ++ & ++ & ++ & ++ & ++ & ++ & ++ & ++ & $\begin{array}{c}\text { Tettamanti et } \\
\text { al. (1997) }\end{array}$ \\
\hline $201 Y$ & $56 \mathrm{D}$ & ++ & ++ & + & & & ++ & & + & + & ++ & & + & + & ++ & & $\begin{array}{c}\text { Yang et al. } \\
\text { (1995) }\end{array}$ \\
\hline $203 Y$ & $1 C$ & + & & + & + & + & ++ & & ++ & ++ & ++ & & ++ & ++ & ++ & & $\begin{array}{c}\text { Tettamanti et } \\
\text { al. (1997) }\end{array}$ \\
\hline $238 Y$ & $48 \mathrm{C}$ & ++ & ++ & ++ & + & + & ++ & ++ & ++ & ++ & ++ & ++ & ++ & ++ & ++ & ++ & $\begin{array}{c}\text { Yang et al. } \\
\text { (1995) }\end{array}$ \\
\hline$c 492 b$ & $49 C$ & & & & & & & & & & & & ++ & ++ & ++ & & this study \\
\hline c739 & $40 \mathrm{~A}$ & ++ & + & + & + & + & ++ & & ++ & ++ & + & & ++ & ++ & & & $\begin{array}{l}\text { Yang et al. } \\
\text { (1995) }\end{array}$ \\
\hline c772 & $42 \mathrm{~A}$ & & & & & & & & ++ & ++ & ++ & ++ & ++ & ++ & ++ & ++ & $\begin{array}{l}\text { Yang et al. } \\
\text { (1995) }\end{array}$ \\
\hline
\end{tabular}

Summary of expression patterns from late third instar larval stage to adulthood. (++) Extensive staining; $(+)$ more restricted staining. Abrupt onset of reporter expression in lines 103Y, c492b, and c772 was interpreted as a temporal change in enhancer activity. $\gamma$ lobe staining in line $c 739$ was lost by the time of eclosion. $\gamma$ lobe staining in line $82 Y$ is not always present.

a further $8 \mathrm{hr}$. A recognizable $\delta$ lobe appears at the same time as the $\gamma$ lobe, and only in lines in which we see persistent staining of $\alpha^{L}$ (30Y, 117Y, and 238Y), with which it may thus be developmentally continuous. Axons of the $\delta$ lobe appear to wrap around the newly forming $\alpha$ lobe (Fig. 6; fourth column, line 30Y, 117Y, and 238Y).

Staining corresponding to the adult $\alpha$ and $\beta$ lobes usually appears just after the formation of the $\gamma$ and $\delta$ lobes, becoming complete at $\sim 48 \mathrm{hr}$ APF or later. We have found no evidence for the adult $\alpha$ and $\beta$ lobes being developmentally continuous with earlier structures. Appearance of $\alpha$ and $\beta$ staining is accompanied by the first appearance of tracts internal to the pedunculus. It is thus possible that the $\alpha$ and $\beta$ lobes are adult-specific structures.

\section{OTHER ELEMENTS OF THE OLFACTORY SYSTEM}

Given the results of Stocker et al. (1997) we were surprised to find that $\mathrm{HU}$ ablation did not dramatically alter the projection patterns of at least tw o classes of MB extrinsic neurons (putative input via the AGT and putative $\gamma$-lobe exit fibers). We did, however, observe effects of ablation on the $A L$, from which the AGT emanates. In particular, many $P\{G A L 4\}$ lines that reveal the adult $\alpha$ and $\beta$ lobes also reveal AL local interneurons that are ablated by HU. Such neurons must therefore arise from, or be dependent upon, the single LNb per hemisphere that is active during the period of $\mathrm{HU}$ treatment (Ito and Hotta 1992). It may be that most or all of the intrinsic neurons of the adult AL arise from just this neuroblast, ablation of which causes a $32 \%$ reduction in AL volume (de Belle and Heisenberg 1994). Projection patterns of AL afferents and efferents appeared largely unaffected by ablation, as did what could be visualized of the glomerular architecture of the AL (cf. Stocker et al. 1997). That HU-ablated flies exhibit olfactory defects (de Belle and Heisenberg 1994) may thus be a specific consequence of the loss of AL intrinsic neurons.

The intrinsic neuronal components of the MBs and the ALs are morphologically different; in the 
former organized as long parallel fiber tracts and in the latter ramifying widely between the various glomeruli. Nevertheless, they would appear to exhibit developmental parallels in the timing of neuroblast activity (Truman and Bate 1988; Prokop and Technau 1991; Ito and Hotta 1992) and a degree of commonalty in terms of their patterns of gene expression (see above).

Although the primary roles of AL and MB neuropils appear different, the above commonalties may hint at a physiological and thus functional relationship between the two classes of intrinsic neurons. Morphological differences may be more a matter of context than identity.

\section{LARVAL AND ADULT MUSHROOM BODY FUNCTION}

The striking differences at the levels of neuron trajectory, gene expression and developmental history betw een KCs that comprise the $\alpha / \beta$ lobes and those of the $\gamma / \delta$ lobes are highly suggestive of different functional roles.Though not yet demonstrated, it seems reasonable to suppose that larval olfactory learning, like adult learning, involves the MBs. Furthermore, memory retention through metamorphosis implies a developmentally continuous structure. We suggest the $\gamma$ and $\delta$ lobes store information of relevance to both developmental stages, whereas the $\alpha$ and $\beta$ lobes have uniquely adult roles, for example, in courtship behavior (see O'Dell et al. 1995).

\section{Acknowledgments}

This work was supported by the UK Biotechnology and Biological Science Research Council (J.D.A., K.K.), the Wellcome Trust (K.K.), and by the Max-Plank-Gesellschaft (J.S.d.B.).

The publication costs of this article were defrayed in part by payment of page charges. This article must therefore be hereby marked "advertisement" in accordance with 18 USC section 1734 solely to indicate this fact.

\section{References}

Aceves-Pina, A.E. and W.G. Q uinn. 1979. Learning in normal and mutant Drosophila larvae. Science 206: 93-96.

Armstrong, J.D. and K. Kaiser. 1997. The study of Drosophila brain development. In Transgenic animals-Generation and use (ed. L.M. Houdebine), pp. 365-370. Harwood Academic Publishers.

Brand, A. and N. Perrimon. 1993. Targeted gene expression as a means of altering cell fates and generating dominant phenotypes. Development 118: 401-415.
Brotz, T. M., B. Bochenek, K. Aronstein, R. H. ffrench-Constant, and A. Borst. 1996. $\gamma$ aminobutyric acid receptor distribution in the mushroom bodies of a fly (Calliphora erythrocephala): A functional subdivision of Kenyon cells? J. Comp. Neurol. 383: 42-48.

Cambiazo, V., T. Tully, and L. Krase. 1994. Memory through metamorphosis in normal and mutant Drosophila. J. Neurosci. 14: 68-74.

Connolly, J.B., I.J.H. Roberts, J.D. Armstrong, K. Kaiser, M. Forte, T. Tully, and C.J. O 'Kane. 1996. Associative learning and memory is disrupted by impaired G(S) signalling in Drosophila mushroom bodies. Science 274: 2104-2107.

de Belle, J.S. and M. Heisenberg. 1994. Associative odor learning in Drosophila is abolished by chemical ablation of mushroom bodies. Science 236: 692-695.

de Belle, J.S. and M. Heisenberg. 1996. Expression of Drosophila mushroom body mutations in alternative genetic backgrounds: A case study of the mushroom body miniature gene (mbm). Proc. Natl. Acad. Sci. 93: 9875-9880.

Goodwin, S.F., M. del Vecchio, S.R.H. Russell, T. Tully, and K. Kaiser. 1997. Defective learning in mutants of the Drosophila gene for a regulatory subunit of CAMP-dependent protein kinase. J. Neurosci. 17: 8817-8827.

Grotewiel, M.S., C.D.O. Beck, K.H. Wu, X.R. Zhu, and R.L. Davis. 1998. Integrin-mediated short-term memory in Drosophila. Nature 391: 455-460.

Han, P.-L., L.R. Levin, R.R. Reed, and R.L. Davis. 1992. Preferential expression of the Drosophila rutabaga gene in mushroom bodies, neural centers for learning in insects. Neuron 9: 619-627.

Hawkins, R.D., E.R. Kandel, and S.A. Siegelbaum. 1993. Learning to modulate transmitter release: Themes and variations in synaptic plasticity. Annu. Rev. Neurosci. 16: $625-665$.

Heisenberg, M. 1989. Genetic approach to learning and memory (mnemogenetics) in Drosophila melanogaster. In Fundamentals of memory formation: Neuronal plasticity and brain function (ed. B. Rahmann), pp. 3-45. Gustav Fischer Verlag, Stuttgart, Germany.

Heisenberg, M., A. Borst, S. Wagner, and D. Byers. 1985. Drosophila mushroom body mutants are deficient in olfactory learning. J. Neurogenet. 2: 1-30.

Heisenberg, M., M. Heusipp, and C. W anke. 1995. Structural plasticity in the Drosophila brain. J. Neurosci.

15: 1951-1960.

Ito, K. and Y. Hotta. 1992. Proliferation pattern of postembyonic neuroblasts in the brain of Drosophila melanogaster. Dev. Biol. 149: 134-148.

Ito, K., W. Awano, K. Suzuki, Y. Hiromi, and D. Yamamoto. 1997. The Drosophila mushroom body is a quadruple

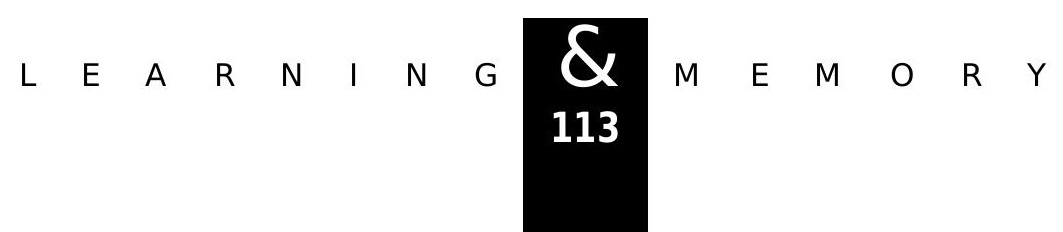




\section{Armstrong et al.}

structure of clonal units each of which contains a virtually identical set of neurones and glial cells. Development 124: 761-771.

Jellies, J.A. 1981. "Associative olfactory conditioning in Drosophila melanogaster and memory retention through metamorphosis." M.Sc. thesis, Illinois State U niversity, N ormal, IL.

Manning, A. 1967. Pre-imaginal conditioning in Drosophila. Nature 216: 338-340.

Nighorn, A., M.J. Healy, and R.L. Davis. 1991. The cyclic AMP phosphodiesterase encoded by the Drosophila dunce gene is concentrated in the mushroom body neuropil. Neuron 6: 455-467.

O 'D ell, K.M.C., J.D. Armstrong, M.Y. Yang, and K. Kaiser. 1995. Functional dissection of the Drosophila mushroom bodies by selective feminisation of genetically defined sub-compartments. Neuron 15: 55-61.

Power, M.E. 1943. The brain of Drosophila melanogster. J. Morphol. 72: 517-559.

Prokop, A. and G.M. Technau. 1991. The origin of postembryonic neuroblasts in the ventral nerve cord of Drosophila melanogaster. Development 111: 79-88.

Prokop, A. and G.M. Technau. 1994. Normal function of the mushroom body defect gene of Drosophila is required for the regulation of the number and proliferation of neuroblasts. Devel. Biol. 161: 321-337.

Skoulakis, E.M.C. and R.L. Davis. 1996. Olfactory learning-deficits in mutants for Leonardo, a Drosophila gene encoding a 14-3-3-protein. Neuron 17: 931-944.

Skoulakis, E.M.C., D. Kalderon, and R.L. Davis. 1993. Preferential expression in the mushroom bodies of the catalytic subunit of protein kinase $A$ and its role in learning and memory. Neuron 11: 197-208.

Stocker, R.F. 1994. The organisation of the chemosensory system in Drosophila melanogaster: A review. Cell Tissue Res. 275: 3-26.

Stocker, R.F., G. Heimbeck, N. Gendre, and J.S. de Belle. 1997. Neuroblast ablation in Drosophila $P\{G A L 4\}$ lines reveals origins of olfactory interneurons. J. Neurobiol. 32: 443-456.

Technau, G.M. 1984. Fiber number in the mushroom bodies of adult Drosophila melanogaster depends on age, sex and experience. J. Neurogenet. 1: 113-126.

Technau, G.M. and M. Heisenberg. 1982. Neural reorganisation during metamorphosis of the corpora pedunculata in Drosophila melanogaster. Nature 295: 405-407.

Tettamanti, M., J.D. Armstrong, K. Endo, M.Y. Yang, K. Furokubo-Tokunaga, K. Kaiser, and H. Reichert. 1997. Early development of the Drosophila mushroom bodies, brain centres for associative learning and memory. Dev. Gene Evol. 207: 242-252.

Truman, J.W . and M. Bate. 1988. Spatial and temporal patterns of neurogenesis in the central nervous system of Drosophila melanogaster. Dev. Biol. 125: 145-157.

Yang, M.Y., J.D. Armstrong, I. Vilinsky, N.J. Strausfeld, and K. Kaiser. 1995. Subdivision of the Drosophila mushroom bodies by enhancer-trap expression patterns. Neuron 15: 45-54.

Received December 2, 1997; accepted in revised form April 24, 1998. 


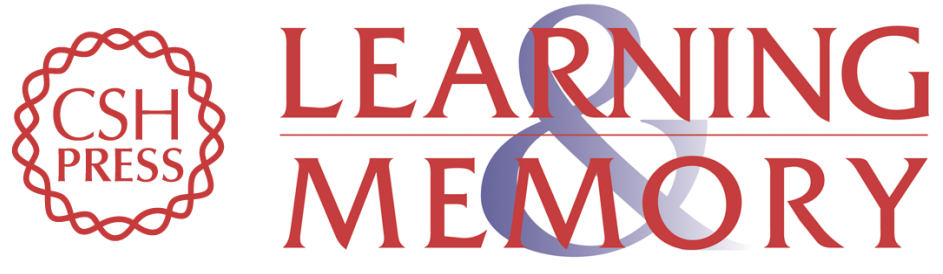

\section{Metamorphosis of the Mushroom Bodies; Large-Scale Rearrangements of the Neural Substrates for Associative Learning and Memory in Drosophila}

J. Douglas Armstrong, J. Steven de Belle, Zongsheng Wang, et al.

Learn. Mem. 1998, 5:

Access the most recent version at doi:10.1101//m.5.1.102

References This article cites 30 articles, 9 of which can be accessed free at: http://learnmem.cshlp.org/content/5/1/102.full.html\#ref-list-1

License

Email Alerting

Receive free email alerts when new articles cite this article - sign up in the box at the Service top right corner of the article or click here. 